This document is the accepted manuscript version of a published work that appeared in final form in Nano letters, copyright ( ) American Chemical Society after peer review and technical editing by the publisher.

To access the final edited and published work see: https://dx.doi.org/10.1021/acs.nanolett.5b01021.

This version has been published under a "All rights reserved" license. 


\section{Mechanical Tuning of LaAlO3/SrTiO3 Interface Conductivity}

P. Sharma, ${ }^{\dagger}$ S. Ryu,$^{\dagger}$ J. D. Burton,,$^{\dagger}$ T. R. Paudel,,$^{\dagger}$ C. W. Bark, ${ }^{\dagger}$ E. Y. Tsymbal, ${ }^{\dagger}$ G. Catalan, ${ }^{\xi^{*}}$ C. B. Eom, ${ }^{+}$and A. Gruverman ${ }^{* *}$

${ }^{\dagger}$ Department of Physics and Astronomy \& Nebraska Center for Materials and Nanoscience, University of Nebraska-Lincoln, NE 68588, USA

${ }^{*}$ Department of Materials Science and Engineering, University of Wisconsin-Madison, WI 53706, USA

${ }^{\S}$ ICREA-Institucio Catalana de Recerca i Estudis Avançats, and ICN2-Institut Catala de Nanociencia i Nanotecnology (Severo Ochoa Excellence Institute), Campus de Bellaterra, Barcelona, Spain

Keywords: Complex oxides, heterointerfaces, 2D electron gas, memristors, resistive switching

In recent years, complex-oxide heterostructures and their interfaces have become the focus of significant research activity, primarily driven by the discovery of emerging states and functionalities that open up opportunities for the development of new oxide-based nanoelectronic devices. The highly conductive state at the interface between insulators $\mathrm{LaAlO}_{3}$ and $\mathrm{SrTiO}_{3}$ is a prime example of such emergent functionality, with potential application in high electron density transistors. In this report, we demonstrate a new paradigm for voltage-free tuning of $\mathrm{LaAlO}_{3} / \mathrm{SrTiO}_{3}$ (LAO/STO) interface conductivity, which involves the mechanical gating of interface conductance through stress exerted by the tip of a scanning probe microscope. The mechanical control of channel conductivity

\footnotetext{
*E-mail: gcatalan@cin2.es

** E-mail: agruverman2@unl.edu
} 
results in transistor functionality with zero gate voltage, and the long retention time of the induced resistance states also enables the use of LAO/STO heterostructures as mechanically-operated memristors.

The discovery of a conducting two-dimensional electron gas (2DEG) at the interface between two band insulators, $\mathrm{SrTiO}_{3}$ and $\mathrm{LaAlO}_{3}{ }^{1}$, has been the subject of intense scrutiny over the past decade. Hypotheses for its origin rank from a "polar catastrophe" generated by the build up of electrostatic potential in the $\mathrm{LaAlO}_{3}$ layer, ${ }^{2}$ to oxygen vacancy accumulation at the interface, ${ }^{3}$ to chemical inter-mixing, ${ }^{4}$ or to a combination of all these factors. Irrespective of its physical origin, the 2DEG at the $\mathrm{LaAlO}_{3} / \mathrm{SrTiO}_{3}$ interface is an interesting functional entity with a wide variety of physical properties, ${ }^{5,6}$ and high potential for applications in oxide electronics. ${ }^{7,8,9,10,11}$ Recent works, ${ }^{8,11,12,13}$ show that the conduction at the $\mathrm{LaAlO}_{3} / \mathrm{SrTiO}_{3}$ interface can be switched between metallic and insulating states by an external voltage using a tip of a scanning probe microscope (SPM) as the gate, thereby replicating the functionality of a field-effect transistor.

In parallel with these investigations of the $\mathrm{LaAlO}_{3} / \mathrm{SrTiO}_{3}$ interface, the physical properties of the $\mathrm{LaAlO}_{3}$ layer itself have also been studied, and an unexpected ferroelectric-like behavior has been reported. ${ }^{14}$ Piezoresponse force microscopy (PFM) studies show that application of an external voltage to ultrathin films of $\mathrm{LaAlO}_{3}$ results in an induced switchable polarization and hysteretic electromechanical response much like the piezoelectric effect in a ferroelectric. Since $\mathrm{LaAlO}_{3}$ is ferroelastic but not ferroelectric in bulk, this effect is rather surprising: although thin films of nonpolar materials can become polar due to epitaxial strain imposed by the substrate, ${ }^{15}$ firstprinciple calculations do not predict the appearance of ferroelectricity in $\mathrm{LaAlO}_{3}$ even under severe epitaxial strain. ${ }^{16}$ It should be noted that the ferroelectric-like state in 
$\mathrm{LaAlO}_{3}$ relaxes slowly over time with a response being reminiscent to that of electrets, where polarization is induced by a transient separation of ionic charge, rather than by a stable long-range dipolar orientation. ${ }^{17,18}$ Accordingly, the switchable polarization has been attributed to the motion of charged oxygen vacancies through the LAO layer under an applied electric field. ${ }^{14}$ From the applied point of view $\mathrm{LaAlO}_{3}$ fully mimics the functionality of true ferroelectrics in terms of both input (excitation field) and output (functional response): the field-induced polarization of $\mathrm{LaAlO}_{3}$ behaves like a ferroelectric in terms of electromechanical response as measured by PFM and ability to tune conductivity ${ }^{14}$ like in ferroelectric field effect transistors (FE-FET). ${ }^{19,20,21,2223}$

Application of external voltage, however, is not the only way to manipulate polarization: it has recently been shown that flexoelectricity (the coupling between polarization and strain gradients) also allows the polarization of ferroelectrics to be switched by mechanical force. ${ }^{24}$ This brings up the enticing possibility of a mechanical control of the polar state of $\mathrm{LaAlO}_{3}$ and, thus, the two-dimensional electron gas (2DEG) at the interface with $\mathrm{SrTiO}_{3}$. In this work, we report such a mechanical gating of the interface conductance driven by the mechanical stress induced by an SPM tip in contact with the $\mathrm{LaAlO}_{3} / \mathrm{SrTiO}_{3}$. This discovery enables transistor functionality at zero gate voltage and provides a new paradigm for voltage-free control of conductivity in oxide heterostructures, opening a route around the current gate-voltage bottleneck in transistor scaling. ${ }^{25}$ In addition, the long retention of stress-tunable interface conductance enables devices that may be termed as mechanically-operated memristors (MOMs).

The epitaxial $\mathrm{LaAlO}_{3} / \mathrm{SrTiO}_{3}$ thin film heterostructures have been grown by pulsed laser deposition on (001) $\left(\mathrm{LaAlO}_{3}\right)_{0.3}-\left(\mathrm{Sr}_{2} \mathrm{AlTaO}_{3}\right)_{0.7}$ (LSAT) substrates (see Supplementary Information for further details). First, by using the $\mathrm{LaAlO}_{3}$ and $\mathrm{SrTiO}_{3}$ layers of 20 and 50 unit cells (u.c.), respectively, we fabricated a conducting 
$\mathrm{LaAlO}_{3} / \mathrm{SrTiO}_{3}$ interface that exhibited a room-temperature conductance value consistent with the previous studies. ${ }^{26}$ Next, we performed electrical writing of polarization using an electrically-biased SPM tip. Figures $1(a, b)$ show an example of the electrically written polarization states in the $\mathrm{LaAlO}_{3} / \mathrm{SrTiO}_{3}$ heterostructures. Detailed analysis shows that this polarization stems from the $\mathrm{LaAlO}_{3}$ layer rather than the $\mathrm{SrTiO}_{3}$ (See Fig. S1 in Supplementary Information). ${ }^{14}$ The PFM patterns of these states are identical to those which would be expected from a true ferroelectric film: namely, there is a $180^{\circ}$ phase difference between regions written with a positive and negative voltage, while the PFM amplitude is exactly zero at the boundary between these regions. Spectroscopic PFM measurements of the electromechanical response as a function of the external bias also show hysteretic behavior (Fig. 1(c)). The switching behavior thus mimics that of a ferroelectric with $180^{\circ}$ domains.

Next, we investigated the effect of mechanical tip pressure on the induced polarization in $\mathrm{LaAlO}_{3} / \mathrm{SrTiO}_{3}$ heterostructures to see if it is possible to invert the polarization mechanically, as recently demonstrated for thin films of the conventional ferroelectric $\mathrm{BaTiO}_{3}{ }^{24} \mathrm{~A} 1 \times 1 \mu \mathrm{m}^{2}$ region of the electrically pre-patterned polarization was scanned with a grounded SPM tip under an incrementally increasing mechanical load (from $100 \mathrm{nN}$ up to $1000 \mathrm{nN}$ ). The PFM images of the resulting states (Figs. 1(d,e)) show mechanically-induced switching of polarization in the left part (poled by $-6 \mathrm{~V})$, which occurs when a mechanical load exceeds $500 \mathrm{nN}$. There is no visible damage to the sample surface as a result of scanning under this loading force (Fig. 1(f)). This result demonstrates that the field-induced polarization in $\mathrm{LaAlO}_{3}$ can be reversed by pure mechanical stress.

The next step is to see whether the mechanically-induced polarization reversal can also be used to modulate the carrier density and, thus, gate the conductivity of the 
$\mathrm{LaAlO}_{3} / \mathrm{SrTiO}_{3}$ interface. The results, presented in Fig. 2, indicate that the answer is positive. Measurements were carried out using a $40 \times 20 \mu \mathrm{m}^{2} \mathrm{LaAlO}_{3} / \mathrm{SrTiO}_{3}$ bridge structure fabricated on the LSAT substrate by ion milling (Fig. 2(a)). Prior to the mechanical test, the conventional electrostatic gating was tested by applying successively $+9 \mathrm{~V}$ and afterward $-9 \mathrm{~V}$ using the SPM tip (Fig. 2(a)). This voltage causes resistance to change by a factor of 2.5 (Fig. 2(b)), in agreement with previously reported results. ${ }^{14}$ Once the interface is set into the high resistance state (by poling with a $-9 \mathrm{~V}$ bias), the effect of the mechanical stress was probed by scanning the bridge area with a grounded PFM tip while applying a variable loading force. As seen in Fig. 2(c), the interface resistance progressively decreases with increasing the mechanical load, thus demonstrating purely mechanical gating of interface conductance.

Figure 2(c) shows that the mechanical load affects only the high resistance states and has an insignificant effect on the low resistance state generated by poling with a +9 $\mathrm{V}$ bias. The mechanically-induced resistance states are still responsive to an external electrical stimulus: application of electrical bias of any polarity drives the resistance up or down to any intermediate level between the low and high resistance states. Mechanically-induced resistance states did not change for at least several hours, indicating a long retention behavior and thus piezo-memristive functionality.

The obtained results indicate that mechanical modulation is fully analogous to the electrostatic one: whatever can be achieved with a voltage, it can also be done with pressure. From the viewpoint of resistance control, the $2 \mu \mathrm{N}$ loading force is equivalent to an electrical bias of $+9 \mathrm{~V}$ (Fig. 2(c)). The same equivalence can be obtained from switching of polarization (Fig. 1(d,e)) and coercive bias (Fig. 1(c)): polarization can be reversed either by a mechanical load above $0.5 \mu \mathrm{N}$ or by an electrical bias above $+2 \mathrm{~V}$. Within error, these two measurements are self-consistent and give an approximate 
voltage/stress equivalence constant of $\gamma \sim 4.5 \mathrm{~V} / \mu \mathrm{N}$. This equivalence is of the same order of magnitude as that of $\mathrm{BaTiO}_{3}$ thin films ${ }^{24}(\gamma \sim 1.8 \mathrm{~V} / \mu \mathrm{N})$, where the driving force for switching is thought to be flexoelectricity.

We interpret the observed switchable PFM response and the memristive functionality of the $\mathrm{LaAlO}_{3} / \mathrm{SrTiO}_{3}$ heterostructures in terms of oxygen vacancy migration driven by mechanical load (inset in Fig 2(a)). Although $\mathrm{LaAlO}_{3}$ is non-polar in the bulk phase, epitaxial deposition of the $\mathrm{LaAlO}_{3}$ on the $\mathrm{TiO}_{2}$ terminated $\mathrm{SrTiO}_{3}$ leads to a polar $\mathrm{LaAlO}_{3}$ film due to a negative ionic charge of the top $\left(\mathrm{AlO}_{2}\right)^{1-}$ monolayer and a positive ionic charge of the interfacial $(\mathrm{LaO})^{1+}$ monolayer. This polar state is energetically unfavorable and is compensated by the accumulation of electrons at the $\mathrm{LaAlO}_{3} / \mathrm{SrTiO}_{3}$ interface and positive charge on the surface of $\mathrm{LaAlO}_{3}$ through oxygen vacancies formation. ${ }^{27,28}$ First-principles density-functional calculations (see Supplementary Information for details) show that the associated energy profile for oxygen vacancy formation has a barrier at the $\mathrm{LaAlO}_{3} / \mathrm{SrTiO}_{3}$ interface with a global minimum at the $\mathrm{LaAlO}_{3}$ surface and a shallow local minimum inside $\mathrm{SrTiO}_{3}$ just below the interface (Fig 2(e)). The presence of this barrier is caused by an intrinsic electric field pointing away from the $(\mathrm{LaO})^{+}$terminated interface layer. Oxygen vacancies are mobile and amenable to migration, which can be induced by an external stimulus. Application of an electrical field causes reversible movement of oxygen vacancies across the barrier at the $\mathrm{LaAlO}_{3} / \mathrm{SrTiO}_{3}$ interface producing the observed switchable piezoresponse behavior in the $\mathrm{LaAlO}_{3} / \mathrm{SrTiO}_{3}$ system. The changing oxygen vacancy distribution alters the electron density at the interface and thus the interface resistance.

We modelled the effect of the oxygen vacancy migration across the $\mathrm{LaAlO}_{3} / \mathrm{SrTiO}_{3}$ interface by solving the drift-diffusion equations (details are given in Supplementary Information and Fig. S2). Starting from the situation when all oxygen 
vacancies are accumulated near the $\mathrm{LaAlO}_{3}$ top surface, which corresponds to the polarization state generated by the negatively biased tip, we considered application of a positive bias of $+9 \mathrm{~V}$, which is equivalent to a loading force of $2 \mu \mathrm{N}$ (Fig. 2(b-c)). This drives the oxygen vacancies out of the $\mathrm{LaAlO}_{3}$ and into the $\mathrm{SrTiO}_{3}$ (Fig. 2(d)), thereby increasing the total density of electrons in the $\mathrm{SrTiO}_{3}$ by a factor of $\sim 2$. This density stays constant for some time after the applied bias has been removed, although the vacancies begin to gradually transfer back to the $\mathrm{LaAlO}_{3}$ surface, which eventually restores the initial state. This long-lived non-equilibrium distribution of oxygen vacancies, and the associated change in the electron density can account for the change in the interface resistance after application of mechanical stress.

The oxygen vacancy migration can be induced either by applying an electric field or, as we report here, by imposing a mechanical stress using the PFM tip. The mechanical biasing may be attributed to the flexoelectric field arising from the tipinduced stress gradient. ${ }^{29}$ Alternatively (or additionally) to the flexoelectric field, oxygen vacancies may also be pushed away from the $\mathrm{LaAlO}_{3}$ surface due to the Vegard effect: since oxygen vacancies generally cause an expansion of the perovskite unit cell volume, ${ }^{30}$ a tip-induced compression should drive them away from the tip-surface contact point. This mechanism, termed piezochemical oxidation, is known to increase the resistivity of $\mathrm{NiO}$ by restoring its full oxygen stoichiometry. ${ }^{31}$ In our case, the sign of the resistance switching is opposite, because the consequence of pushing oxygen vacancies away from the tip is to drive them towards the $\mathrm{LaAlO}_{3} / \mathrm{SrTiO}_{3}$ interface, which results in higher electron density and thus higher conductivity/lower resistivity.

The present work demonstrates all-oxide three-terminal nanodevices with memristive functionality, activated not by voltage but by mechanical pressure -what one mignt call mechanically-operated memristors. The ability to store and process 
information makes such mechanically-operated memristors well-positioned for application in low-power logic devices and neuromorphic circuits. The mechanical gating of oxide interfacial conductivity also opens a new - and much-needed ${ }^{25}$ - path towards transistors whose performance is not limited by gate voltage. 


\section{Figure Captions}

Figure 1. Electrical and mechanical switching of polarization in $\mathrm{LaAlO}_{3} / \mathrm{SrTiO}_{3}$ heterostructures. After electrical pre-poling $( \pm 6 \mathrm{~V})$ in to a bi-domain state (a) PFM amplitude and, corresponding (b) PFM phase image respectively. (c) Local spectroscopic PFM amplitude and phase hysteresis loops acquired at a fixed location on the $\mathrm{LaAlO}_{3}$ surface. After mechanical writing (d) PFM amplitude and, corresponding (e) PFM phase image respectively. Dotted rectangle in $(\mathrm{d}, \mathrm{e})$ marks the area where incrementally increasing loading force $(0.1 \mu \mathrm{N}$ to $1.0 \mu \mathrm{N}$ in steps of $0.1 \mu \mathrm{N})$ was applied. (f) Topography image of the $\mathrm{LaAlO}_{3}$ surface after mechanical writing.

\section{Figure 2. Mechanical tuning of resistivity of $2 \mathrm{DEG}$ in the $\mathrm{LaAlO}_{3} / \mathrm{SrTiO}_{3}$} heterostructures. (a) A schematic view of the structure used for investigation of the tip-induced stress effect on 2DEG conductivity. Inset illustrates redistribution of oxygen vacancies under the applied stress. (b) Bias dependence of the interface resistance as a function of mechanical load exerted by a grounded AFM probe. (c) Interface resistance as a function of mechanical load for both the high (after application of the $-9 \mathrm{~V}$ bias) and the low (after application of the $+9 \mathrm{~V}$ bias) resistance states. (d) Density distribution of oxygen vacancies ( $N_{V}$, solid curves) and electrons ( $n_{e}$, dashed curves) across the $\mathrm{LaAlO}_{3} / \mathrm{SrTiO}_{3}$ interface before and after application of the $+9 \mathrm{~V}$ bias predicted by the drift-diffusion modeling. (e) Oxygen vacancy formation energy profile across the $\mathrm{LaAlO}_{3} / \mathrm{SrTiO}_{3}$ interface calculated within the density functional theory. The lines are guides to eye. 

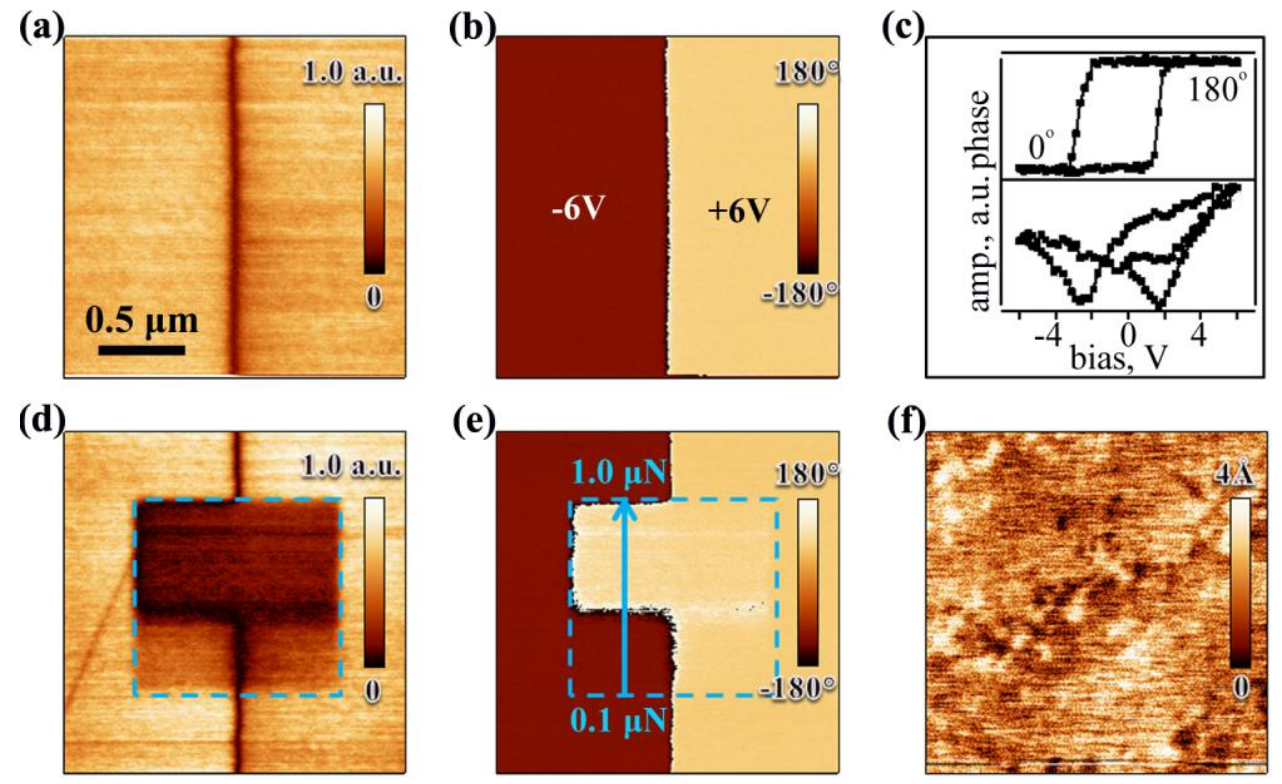

Fig 1 
(a)

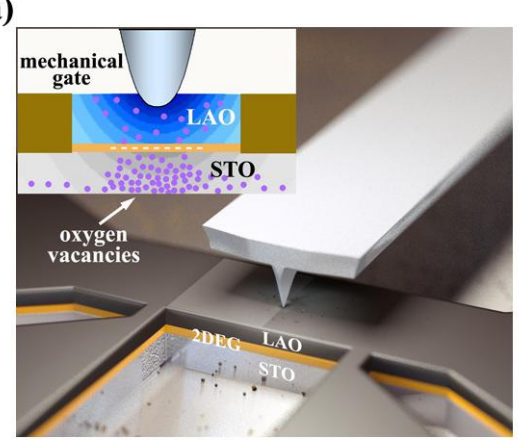

(c)

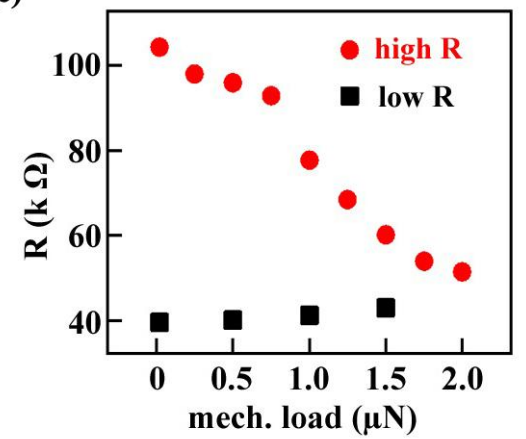

(b)

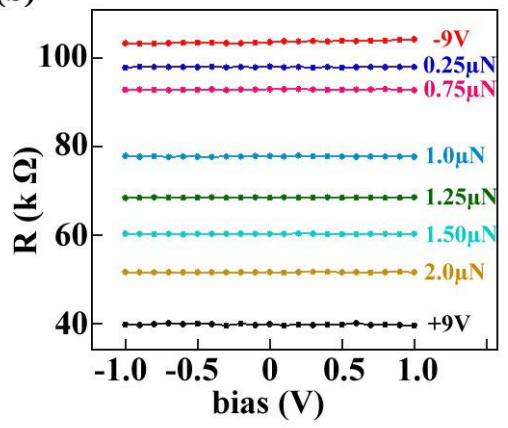

(d)

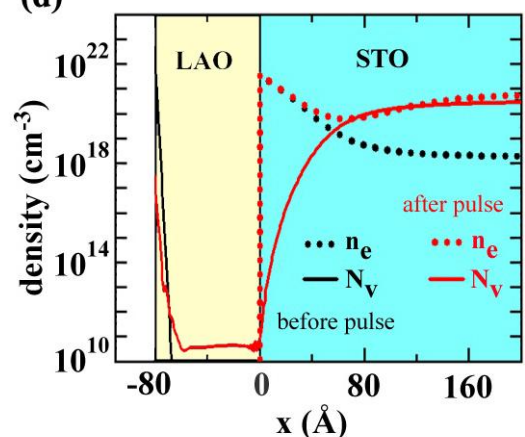

(e)

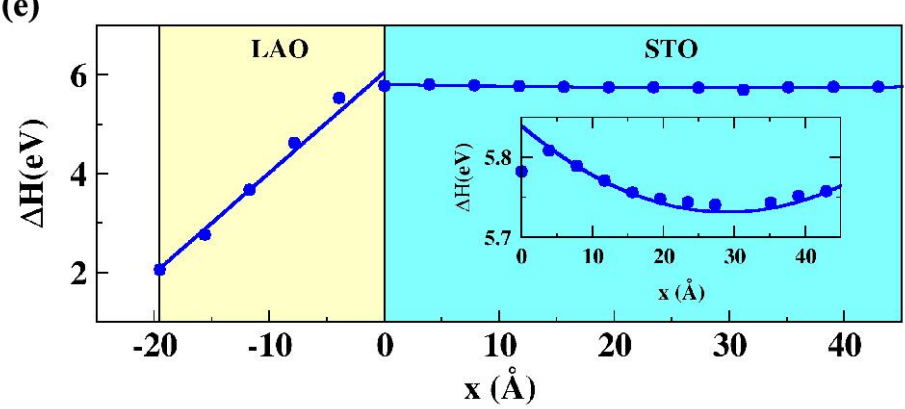

Fig 2 


\section{ASSOCIATED CONTENT}

\section{Supporting Information}

Detailed information on materials and methods, electrical and mechanical switching in $\mathrm{LaAlO}_{3}$ thin films, drift diffusion modelling of oxygen vacancy migration process, and first-principle calculations of the oxygen vacancy formation energy is provided. This material is available free of charge via the Internet at http://pubs.acs.org.

\section{AUTHOR INFORMATION}

\section{Corresponding Author}

Email: agruverman2@unl.edu

\section{Author Contributions}

A. G. and P. S. conceived the idea and designed the experiment. P. S. implemented experimental measurements. S. R. fabricated the samples, C. B. E. supervised sample preparation. J. D. B., T. R. P. and E. Y. T. performed theoretical modelling. A. G. and G. C. analysed the results and wrote the paper. All the authors contributed to the final paper preparation. All authors have given approval to the final version of the manuscript.

\section{ACKNOWLEDGMENT}

This research was supported by the Materials Research Science and Engineering Center (NSF grant DMR-0820521). The work at University of Wisconsin-Madison was supported by the NSF grant DMR-1234096 and the AFOSR grant FA9550-12-1-0342. G. C. acknowledges financial support from an European Research Council (ERC) Starting Grant. First-principles calculations were performed at the University of Nebraska Holland Computing Center and at the Center for Nanophase Materials 
Sciences, which is sponsored at Oak Ridge National Laboratory by the Scientific User Facilities Division, Office of Basic Energy Sciences, U.S. Department of Energy. 3D image of the SPM tip in Fig. 2(a) is courtesy of Dr. Joel Brehm, UNL 


\section{References}

(1) Ohtomo, A.; Hwang, H. Y. Nature 2004, 427, 423.

(2) Nakagawa, N.; Hwang, H. Y.; Muller, D. A. Nat. Mater. 2006, 5, 204.

(3) Herranz, G.; Basletić, M.; Bibes, M.; Carrétéro, C.; Tafra, E.; Jacquet, E.; Bouzehouane, K.; Deranlot, C.; Hamzić, A.; Broto, J.-M.; Barthélémy, A.; Fert, A. Phys. Rev. Lett. 2007, 98, 216803.

(4) Willmott, P. R.; Pauli, S. A.; Herger, R.; Schlepütz, C. M.; Martoccia, D.; Patterson, B. D.; Delley, B.; Clarke, R.; Kumah, D.; Cionca, C.; Yacoby, Y. Phys. Rev. Lett. 2007, 99, 155502.

(5) Brinkman, A.; Huijben, M.; van Zalk, M.; Huijben, J.; Zeitler, U.; Maan, J. C.; van der Wiel, W. G.; Rijnders, G.; Blank, D. H. A.; Hilgenkamp, H. Nat. Mater. 2007, 6, 493.

(6) Li, L.; Richter, C.; Mannhart, J.; Ashoori, R. C. Nat. Phys. 2011, 7, 762.

(7) Mannhart, J.; Schlom, D. G. Science 2010, 327, 1607.

(8) Hwang, H. Y.; Iwasa, Y.; Kawasaki, M.; Keimer, B.; Nagaosa, N.; Tokura, Y. Nat. Mater. 2012, 11, 103.

(9) Cen, C.; Thiel, S.; Mannhart, J.; Levy, J. Science 2009, 323, 1026.

(10) Irvin, P.; Huang, M.; Wong, F. J.; Sanders, T. D.; Suzuki, Y.; Levy, J. Appl. Phys. Lett. 2013, 102, 103113.

(11) Cheng, G.; Siles, P. F.; Bi, F.; Cen, C.; Bogorin, D. F.; Bark, C. W.; Folkman, C. M.; Park, J.-W.; Eom, C.-B.; Medeiros-Ribeiro, G.; Levy, J. Nat. Nanotech. 2011, 6, 343.

(12) Cen, C.; Thiel, S.; Hammerl, G.; Schneider, C. W.; Andersen, K. E.; Hellberg, C. S.; Mannhart, J.; Levy, J. Nat. Mater. 2008, 7, 298. 
(13) Bogorin, D. F.; Bark, C. W.; Jang, H. W.; Cen, C.; Folkman, C. M.; Eom, C.-B.; Levy, J. Appl. Phys. Lett. 2010, 97, 013102.

(14) Bark, C. W.; Sharma, P.; Wang, Y.; Baek, S. H.; Lee, S.; Ryu, S.; Folkman, C. M.; Paudel, T. R.; Kumar, A.; Kalinin, S. V.; Sokolov, A.; Tsymbal, E. Y.; Rzchowski, M. S.; Gruverman, A.; Eom, C. B. Nano Lett. 2012, 12, 1765.

(15) Haeni, J. H.; Irvin, P.; Chang, W.; Uecker, R.; Reiche, P.; Li, Y. L.; Choudhury, S.; Tian, W.; Hawley, M. E.; Craigo, B.; Tagantsev, A. K.; Pan, X. Q.; Streiffer, S. K.; Chen, L. Q.; Kirchoefer, S. W.; Levy, J.; Schlom, D. G. Nature 2004, 430, 758.

(16) Hatt, A. J.; Spaldin, N. A. Phys. Rev. B 2010, 82, 195402.

(17) Jacobs, H. O.; Whitesides, G. M. Science 2001, 291, 1763.

(18) Sessler, G. M. Electrets; Springer: Berlin, 1987.

(19) Ahn, C. H.; Gariglio, S.; Paruch, P.; Tybell, T.; Antognazza, L.; Triscone, J.-M. Science 1999, 284, 1152.

(20) Mathews, S.; Ramesh, R.; Venkatesan, T.; Benedetto, J. Science 1997, 276, 238.

(21) Takahashi, K. S.; Gabay, M.; Jaccard, D.; Shibuya, K.; Ohnishi, T.; Lippmaa, M.; Triscone, J.-M. Nature 2006, 441, 195.

(22) Tra, V. T.; Chen, J.-W.; Huang, P.-C.; Huang, B.-C.; Cao, Y.; Yeh, C.-H.; Liu, H.J.; Eliseev, E. A.; Morozovska, A. N.; Lin, J.-Y.; Chen, Y-C.; Chu, M.-W.; Chiu, P.-W.; Chiu, Y.-P.; Chen, L.-Q.; Wu, C.-L.; Chu, Y.-H. Adv. Mater. 2013, 25, 3357.

(23) Huang, M.; Bi, F.; Ryu, S.; Eom, C.-B.; Irvin, P.; Levy, J. APL Mat. 2013, 1, 052110.

(24) Lu, H.; Bark, C.-W.; Esque de los Ojos, D.; Alcala, J.; Eom, C. B.; Catalan, G.; Gruverman, A. Science 2012, 336, 59.

(25) Theis, T. N.; Solomon, P. M. Science 2010, 327, 1600.

(26) Bark, C. W.; Felker, D. A.; Wang, Y.; Zhang, Y.; Jang, H. W.; Folkman, C. M.; 
Park, J. W; Baek, S. H.; Zhou, H.; Fong, D. D.; Pan, X. Q.; Tsymbal, E. Y.; Rzchowski, M. S.; Eom, C. B. Proc. Natl. Acad. Sci. USA 2011, 108, 4720.

(27) Breckenfeld, E.; Bronn, N.; Karthik, J.; Damodaran, A. R.; Lee, S.; Mason, N.; Martin, L. W. Phys. Rev. Lett. 2013, 110, 196804.

(28) Pavlenko, N.; Kopp, T.; Tsymbal, E. Y.; Sawatzky, G. A.; Mannhart, J. Phys. Rev. B 2012, 85, 020407.

(29) Zubko, P.; Catalan, G.; Tagantsev, A. K. Annu. Rev. Mater. Res. 2013, 43, 387.

(30) Denton, A. R.; Ashcroft, N. W. Phys. Rev. A 1991, 43, 3161.

(31) Kim, Y.; Kelly, S. J.; Morozovska, A.; Rahani, E. K.; Strelcov, E.; Eliseev, E.; Jesse, S.; Biegalski, M. D.; Balke, N.; Benedek, N.; Strukov, D.; Aarts, J.; Hwang, I.; Oh, S.; Choi, J. S.; Choi, T.; Park, B. H.; Shenoy, V. B.; Maksymovych, P.; Kalinin, S. V. Nano Lett. 2013, 13, 4068. 


\section{Table of Content Figure}
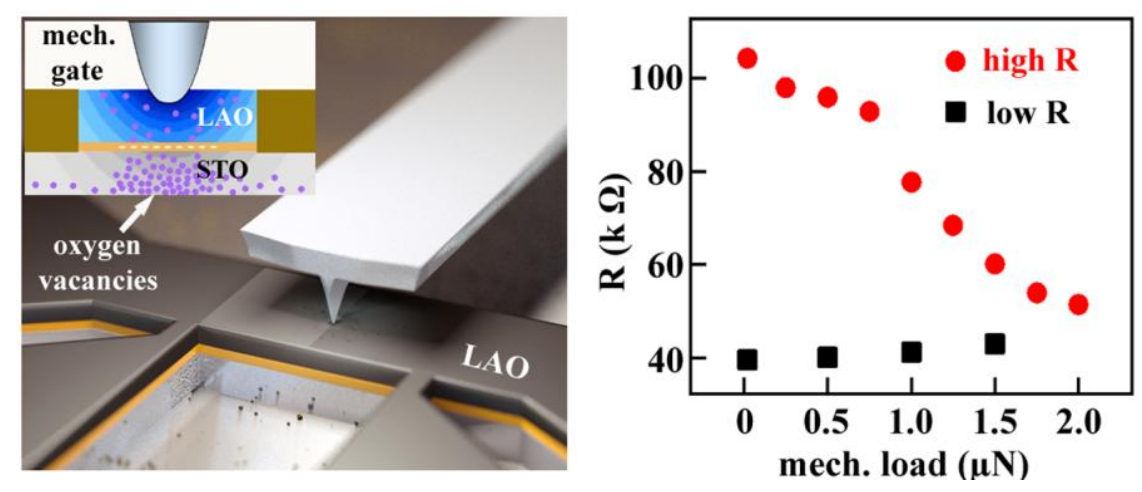

Synopsis: An unexpected and potentially very useful result - a voltage-free tuning of the conductivity of the $\mathrm{LaAlO}_{3} / \mathrm{SrTiO}_{3}$ interface - has been observed. This new functionality is realized via the mechanical gating of interface resistance through mechanical stress, exerted by the tip of a scanning probe microscope. The continuous tunability and the long retention time of the mechanically-induced interfacial conductivity states constitute a type of electronic devices with enhanced functionality that we call mechanically-operated memristors. 